\title{
Theoretical model for cell migration with gradient sensing and shape deformation
}

\author{
Tetsuya Hiraiwa $^{\mathrm{a}}$, Akinori Baba, and Tatsuo Shibatab \\ Center for Developmental Biology, RIKEN, Chuo-ku, Kobe, Hyogo, Japan and \\ Japan Science and Technology Agency, CREST, 1-3 Yamadaoka, Suita, Osaka 565-0871, Japan \\ Received 19 October 2012 and Received in final form 7 December 2012 \\ Published online: 11 April 2013 \\ (C) The Author(s) 2013. This article is published with open access at Springerlink.com
}

\begin{abstract}
Amoeboid cells take various shapes during migration, depending on the cell type and its environment. Deformability of the cell shape can then affect the migrating behavior. In this article, we introduce a theoretical model of chemotactic cell migration with elliptical shape deformation. Based on the model, we calculate the stationary distributions of the migration directions analytically. As a result, we find that the distributions show different characteristics depending on the difference in the interdependence of the internal polarity, cell morphology and gradient sensing.
\end{abstract}

\section{Introduction}

The migration of living cells sheds light on investigations into a wide variety of dynamics in active matter. The eukaryotic cell is one such active material, consisting of Factin and other cytoskeletal structures. Many eukaryotic cells show motile behaviors by deforming their shapes in a random direction, including extensions and contractions. Spontaneous intracellular activities probably induce such cell shape deformations and motile processes $[1,2]$. One of the remarkable characteristics of cell migration is the cell's ability to determine the direction of the stimulation correctly from its strongly fluctuating surroundings, which is a striking contrast to non-living active matter. By the ability to sense a chemical gradient, cells deform their shapes to orient their cell bodies along the gradient to achieve directional cell migration or chemotaxis. The cell motility, together with the sensing of the chemical stimulation, has been studied by combining theoretical and experimental approaches [3].

Quantitative experiments have been carried out extensively for spontaneous and chemotactic motions of Dictyostelium cells [4-9]. These studies show that the cell motion is not a simple random walk, but exhibits a directional persistence with a correlation time of several minutes. This far exceeds the correlation time of cell protrusions into pseudopods, by which amoebic cells are typically thought to move [4]. To explain the characteristics of cell motility, models of persistent $[4,5]$ and biased random motion [10] have been proposed for spontaneous and chemotactic mi-

\footnotetext{
a e-mail: tetsuhiraiwa@gmail.com

b e-mail: tatsuoshibata@cdb.riken.jp
}

gration trajectories, respectively. Following these studies, we consider that, irrespective of isotropic and anisotropic external conditions, eukaryotic cells can maintain their internal polarities, which determine the cell deformations for movement. In fact, some intracellular processes of chemotactic cells have been demonstrated to exhibit a nonuniform spatial distribution under isotropic conditions. Furthermore, even in undeformable cells treated with an F-actin polymerization inhibitor reagent, some intracellular signals, which could induce F-actin formation if the reagent were absent, form a spatially localized pattern inside the cells [11-13].

Therefore, in this paper, we first focus on a cell motility model that couples the spontaneously established internal polarity with the gradient sensing (see sect. 2) and cell morphology dynamics (see sect. 3) without invoking a particular molecular mechanism. We take into account the dependence of gradient sensing on the cell shape, motivated by recent theoretical studies $[14,15]$, as explained in sect. 3. We then perform the numerical analysis of our model and find that the stationary distribution of polarity directions shows a characteristic profile distinct from the circular normal distribution, as observed experimentally [3] (see sect. 4). To explain the numerical result, we derive analytically the distribution of migration directions of deformable cells (see sect. 5). Finally, we give a summary and discussion (see sect. 6).

\section{Model without shape deformation}

We first consider a model of the internal polarity which depends on the directional sensing as illustrated in fig. 1(a). 


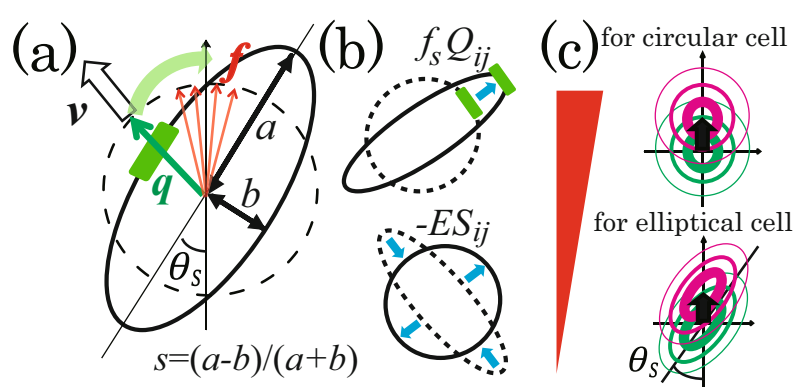

Fig. 1. (Color on-line) Model of cell motility with internal polarity, shape deformation and gradient sensing. (a),(b) Black ellipses indicate the cell shape. (b) The driving force and the restoring force acting on the cell morphology described by the two terms in eq. (12) (blue arrows of upper and lower panels, respectively). (c) The distributions of the coarse-grained driving force $\left\langle\boldsymbol{f}^{0}\right\rangle+\boldsymbol{\xi}^{0}$ and $\langle\boldsymbol{f}\rangle+\boldsymbol{\xi}$ on the internal polarity $\boldsymbol{q}$ for circular and elliptical cells (upper and lower panels), respectively. The probability density functions of $\boldsymbol{\xi}^{0}$ or $\boldsymbol{\xi}$ (indicated as the green contour lines) are shifted (to the magenta contour lines) by $\left\langle\boldsymbol{f}^{0}\right\rangle$ or $\langle\boldsymbol{f}\rangle$ (black arrows) under a shallow chemical gradient.

The magnitude and direction of the internal polarity are described by the vector $\boldsymbol{q}=\left(q_{x}, q_{y}\right)=q\left(-\sin \theta_{q}, \cos \theta_{q}\right)$. Then, the evolution equation for spontaneous polarization is given by

$$
\frac{\mathrm{d}}{\mathrm{d} t} q_{i}=I_{q}\left(1-q^{2}\right) q_{i}+f_{i}^{0} \quad(i=x, y)
$$

In addition to the first term on the right-hand side showing the self-polarization ( $I_{q}$ is a positive constant), we have included the term $\boldsymbol{f}^{0}=\left(f_{x}^{0}, f_{y}^{0}\right)$ describing the driving force to the internal polarity. Here, the direction of $f^{0}$ may be determined by intracellular processes that estimate the gradient direction for migration based on the distribution of chemoattractant-occupied receptors on the surface. (The extracellular gradient is directed to the $y$ axis with $\theta_{q}=0$.) The term $\boldsymbol{f}^{0}$ is thus determined by the estimated gradient direction to which the internal polarity is biased and the responsiveness $f_{q}$ of the internal polarity to the direction, as

$$
\boldsymbol{f}^{0}=f_{q}(-\sin \psi, \cos \psi),
$$

with angle $\psi$ from the $y$-axis to the estimated gradient direction. Because binding and unbinding between chemoattractants and receptors are stochastic processes, the direction $\psi$ is a random variable with an associated probability density function (PDF). The correlation time of $\psi$ is given by the time constant of the chemoattractant-receptor reaction, $\tau_{R} \equiv\left(k_{d}+k_{a} C_{0}\right)^{-1}[3]$, where $C_{0}$ is the chemoattractant concentration and $k_{d}$ and $k_{a}$ are the dissociation and association rates, respectively. In Dictyostelium cells, $\tau_{R}$ is about 1 second $[16,17]$, and hence much smaller than the persistence time $\tau_{P}$ of the migration direction $\approx 300$ seconds [4]. In a reasonable approximation, we suppose that on the time scale of the persistence time $\tau_{P}$, the migration speed $v$ is constant $[3,8]$, and the velocity $\boldsymbol{v}=v\left(-\sin \theta_{v}, \cos \theta_{v}\right)$ immediately follows the direction of the internal polarity $\boldsymbol{q}$, i.e., $\theta_{v}=\theta_{q}$.

On the time scale $\tau_{P} \gg \tau_{R}$, we can apply the central limit theorem to the noisy driving force $\boldsymbol{f}^{0} \approx\left\langle\boldsymbol{f}^{0}\right\rangle+\boldsymbol{\xi}^{0}$ in eq. (1), giving the equation for $\boldsymbol{q}$ as

$$
\frac{\mathrm{d}}{\mathrm{d} t} q_{i}=I_{q}\left(1-q^{2}\right) q_{i}+\left\langle f_{i}^{0}\right\rangle+\xi_{i}^{0} \quad(i=x, y),
$$

where

$$
\left\langle\boldsymbol{f}^{0}\right\rangle=\left(0, f_{q} A^{0} / 2\right)
$$

is the average driving force due to the chemical gradient, and $\boldsymbol{\xi}^{0}$ is white Gaussian noise with $\left\langle\boldsymbol{\xi}^{0}\right\rangle=(0,0)$ and $\left\langle\boldsymbol{\xi}^{0}\left(t^{\prime}\right) \cdot \boldsymbol{\xi}^{0}(t)\right\rangle=2 f_{q}^{2} \tau_{R} \delta\left(t-t^{\prime}\right)$. Here, $A^{0}$ is the amplitude of the bias to the direction of the chemical gradient. Hereafter, we consider the case when $I_{q} \rightarrow \infty$ with the constant strength of the internal polarity $q \rightarrow 1$. Since the relaxation time of $q, \sim I_{q}^{-1}$, is much shorter than that of the polarity direction, $f_{q}^{-1}$, i.e., $I_{q}^{-1} \ll f_{q}^{-1}$, eq. (3) can be rewritten as

$$
\frac{\mathrm{d} \theta_{q}}{\mathrm{~d} t}=-\frac{f_{q} A^{0}}{2} \sin \theta_{q}+\xi_{\perp}^{0}
$$

where $\xi_{\perp}^{0}$ is the component of $\boldsymbol{\xi}^{0}$ perpendicular to the polarity direction $\boldsymbol{q}$. The noise term $\xi_{\perp}^{0}$ satisfies $\left\langle\xi_{\perp}^{0}\right\rangle=0$ and $\left\langle\xi_{\perp}^{0}\left(t^{\prime}\right) \xi_{\perp}^{0}(t)\right\rangle=f_{q}^{2} \tau_{R} \delta\left(t-t^{\prime}\right)$.

From eq. (5), the mean and mean square displacement of $\theta_{q}$ are found to be

$$
\begin{aligned}
\left.\left\langle\theta_{q}(t+\Delta t)-\theta_{q}(t)\right\rangle\right|_{\theta_{q}(t)=\theta_{q}} & =-\frac{f_{q}}{2} A^{0} \sin \theta_{q} \Delta t, \\
\left.\left\langle\left[\theta_{q}(t+\Delta t)-\theta_{q}(t)\right]^{2}\right\rangle\right|_{\theta_{q}(t)=\theta_{q}} & =f_{q}^{2} \tau_{R} \Delta t,
\end{aligned}
$$

and the higher-order moments are $O\left(\Delta t^{2}\right)$. Using the Kramers-Moyal expansion, the time evolution of the PDF $P\left(\theta_{q}, t\right)$ is therefore given by the following Fokker-Planck equation:

$$
\frac{\partial P\left(\theta_{q}, t\right)}{\partial t}=\frac{\partial}{\partial \theta_{q}}\left[c_{q}^{0} P\left(\theta_{q}, t\right)\right]+\frac{\partial^{2}}{\partial \theta_{q}^{2}}\left[D_{q}^{0} P\left(\theta_{q}, t\right)\right],
$$

where $c_{q}^{0}=\left(f_{q} A^{0} \sin \theta_{q}\right) / 2$ and $D_{q}^{0}=\left(f_{q}^{2} \tau_{R}\right) / 2$. This equation has been used in previous works $[3,18]$.

When the chemical gradient is absent $\left(A^{0}=0\right)$, we can use eq. (8) to obtain the correlation time $\tau_{q}$ of the internal polarity and the spontaneous migration as $\tau_{q}=\left(D_{q}^{0}\right)^{-1}=$ $2\left(k_{d}+k_{a} C_{0}\right) / f_{q}^{2}$. When the chemical gradient is present $\left(A^{0} \neq 0\right)$, the stationary distribution is the circular normal distribution $(\mathrm{CN})$

$$
P\left(\theta_{q}\right) \propto \exp \left(\kappa \cos \theta_{q}\right)
$$

where $\kappa \equiv A^{0} /\left(f_{q} \tau_{R}\right)$ is the concentration parameter, which characterizes the inverse of the dispersion. This result is a natural consequence of the central limit theorem for the situation where the noise in the driving force is independent of the migration direction of the circular cell as illustrated in fig. 1(c) top. Hence, the $\mathrm{CN}$ distribution is a robust property, independent of the details of the model as long as the cell takes a circular shape. 


\section{Taking cell shape into account}

Cell shape can affect the statistics of migration and polarity directions. For example, the effect of the shape on the motion of self-propelled objects without gradient sensing has been investigated extensively $[19,20]$. The gradient sensing ability can also be sensitive to the cell shape [21, $14,15]$. Hence, in this article, we focus on the dependence of the gradient sensing on the cell shape, which can have indirect but non-negligible effects on the statistics of polarity and chemotactic migration directions as shown below.

We consider an elliptically deformable cell, as shown in figs. 1(a) and (b). Reflecting the symmetry of the ellipse under the transformation $\boldsymbol{n} \leftrightarrow-\boldsymbol{n}$ with the unit vector $\boldsymbol{n}=\left(-\sin \theta_{s}, \cos \theta_{s}\right)$ parallel to the major (long) axis of the cell, the deformation magnitude and direction can be expressed by the tensor $\boldsymbol{S}$ defined as [22]

$$
S_{i j}=s\left(n_{i} n_{j}-\frac{\delta_{i j}}{2}\right),
$$

where $s=(a-b) /(a+b) \geq 0$ measures the degree of the deformation, and $a$ and $b$ are the lengths of the semi-axes $(a>b)[19,20]$.

The average driving force exerted on the internal polarity, given by $\left\langle\boldsymbol{f}^{0}\right\rangle$ in eq. (3), may be dependent on the cell shape and orientation. It is also expected that, in general, the noise term in the coarse-grained driving force $\boldsymbol{\xi}^{0}$ in eq. (3), can be biased according to the cell shape and orientation, as shown in fig. 1(c). Therefore, the time evolution of the polarity $\boldsymbol{q}$ may be written as

$$
\frac{\mathrm{d} \boldsymbol{q}}{\mathrm{d} t}=I_{q}\left(1-q^{2}\right) \boldsymbol{q}+\langle\boldsymbol{f}(\boldsymbol{S}(t))\rangle+\boldsymbol{\xi}(t, \boldsymbol{S}(t)),
$$

where $\langle\boldsymbol{f}\rangle$ is the averaged driving force, which depends on the morphological factors as $\langle\boldsymbol{f}(\boldsymbol{S})\rangle=\left(0, f_{q} A(\boldsymbol{S}) / 2\right)$, and $\boldsymbol{\xi}(t, \boldsymbol{S})$ is white Gaussian noise with $\langle\boldsymbol{\xi}\rangle=(0,0)$ and $\left\langle\boldsymbol{\xi}\left(t^{\prime}, \boldsymbol{S}\right) \otimes \boldsymbol{\xi}(t, \boldsymbol{S})\right\rangle=2 \boldsymbol{D}_{\boldsymbol{q}}(\boldsymbol{S}) \delta\left(t-t^{\prime}\right)$ with the tensor product $\otimes$. Here, $A(\boldsymbol{S})$ and $\boldsymbol{D}_{\boldsymbol{q}}(\boldsymbol{S})$ are the morphologydependent bias amplitude in the direction estimation and noise strength tensor, respectively. We assume a stochastic integral of Ito type for multiplicative noise in eq. (11). We shall discuss particular forms of the functions $A(\boldsymbol{S})$ and $\boldsymbol{D}_{\boldsymbol{q}}(\boldsymbol{S})$ below. When no driving force acts on the cell deformation, we suppose that an elliptically deformed cell with amplitude $s$ gradually relaxes to a circular shape as $\mathrm{d} s / \mathrm{d} t=-E s$ with an elastic coefficient $E$. Hence, when the driving force deforms the cell elliptically in the direction of the internal polarity $\boldsymbol{q}$, the simplest equation for $S_{i j}$ is $[19,20,23]$

$$
\frac{\mathrm{d}}{\mathrm{d} t} S_{i j}=-E S_{i j}+f_{s} Q_{i j}
$$

where $f_{s}$ and $Q_{i j} \equiv q_{i} q_{j}-\delta_{i j} / 2$ are the strength and direction of the driving force for the deformation, respectively. When $f_{s}$ is positive or negative, the cell elongates its body into the direction parallel or perpendicular to the polarity direction $\boldsymbol{q}$, i.e. migration direction, respectively. Therefore, $f_{s}$ may be positive for a Dictyostelium cell, whereas $f_{s}$ seems to be negative for a fish keratocyte, which is a migrating cell that elongates its body perpendicularly to the migration direction. We note that, in eq. (11), direct coupling terms between $\boldsymbol{q}$ and $\boldsymbol{S}$ are not considered, unlike the previous works for the dynamics of self-propelled particles $[19,20,23]$. This assumption rules out the instability of the straight motion and restricts the cell behavior to rectilinear motion in the absence of noise.

Using the Kramers-Moyal expansion, we obtain the following Fokker-Planck equation for eqs. (11) and (12) with the angular representation as

$$
\begin{aligned}
& \frac{\partial}{\partial t} P\left(\theta_{q}, s, \theta_{s}, t\right)= \\
& \quad \frac{\partial}{\partial \theta_{q}}\left[c_{q} P\left(\theta_{q}, s, \theta_{s}, t\right)\right]+\frac{\partial^{2}}{\partial \theta_{q}^{2}}\left[D_{q} P\left(\theta_{q}, s, \theta_{s}, t\right)\right] \\
& \quad+\frac{\partial}{\partial s}\left[c_{s} P\left(\theta_{q}, s, \theta_{s}, t\right)\right]+\frac{\partial}{\partial \theta_{s}}\left[c_{\theta_{s}} P\left(\theta_{q}, s, \theta_{s}, t\right)\right],
\end{aligned}
$$

where $c_{q}=\left[f_{q} A\left(s, \theta_{s}\right) \sin \theta_{q}\right] / 2, c_{s}=E s-f_{s} \cos 2\left(\theta_{q}-\theta_{s}\right)$, and $c_{\theta_{s}}=-f_{s} \sin 2\left(\theta_{q}-\theta_{s}\right) /(2 s)$. Here, the bias amplitude $A\left(s, \theta_{s}\right)$ in the direction estimation is given by

$$
A\left(s, \theta_{s}\right)=A^{0}\left[1+\Delta_{0}^{A}(s)-\Delta_{1}^{A}(s) \cos 2 \theta_{s}\right],
$$

where $\Delta_{0}^{A}(s)$ and $\Delta_{1}^{A}(s)$ are particular functions satisfying $\Delta_{0}^{A}(0)=\Delta_{1}^{A}(0)=0$. Furthermore, due to the anisotropy in the dispersion of $\boldsymbol{\xi}(t, \boldsymbol{S})$ illustrated in fig. 1(c) bottom, the mean square displacement of $\theta_{q}$ is given by

$$
\left.\left\langle\left[\theta_{q}(t+\Delta t)-\theta_{q}(t)\right]^{2}\right\rangle\right|_{\theta_{q}(t)=\theta_{q}}=2 D_{q}\left(\theta_{q}, s, \theta_{s}\right) \Delta t .
$$

Therefore, the diffusion constant $D_{q}$ depends on the cell orientation $\theta_{s}$ and polarity direction $\theta_{q}$. The dependence of $D_{q}$ on the cell morphology may be written as

$$
D_{q}\left(\theta_{q}, s, \theta_{s}\right)=D_{q}^{0}\left[1+\Delta_{0}^{D}(s)-\Delta_{1}^{D}(s) \cos 2\left(\theta_{q}-\theta_{s}\right)\right] .
$$

The third term on the right-hand side of eq. (16) characterizes the anisotropy in the dispersion of $\boldsymbol{\xi}$ when $s>0$. Here, $\Delta_{0}^{D}(s)$ and $\Delta_{1}^{D}(s)$ are particular functions which satisfy $\Delta_{0}^{D}(0)=\Delta_{1}^{D}(0)=0$. In appendix $\mathrm{A}$, we derive eq. (11) with eqs. (14) and (16). It should be noted that, when $f_{s}<0$, the PDF of polarity directions takes the same form as the case with $f_{s}>0$ by rotating the cell shape by $\pi / 2$ as $\theta_{s} \rightarrow \theta_{s}+\pi / 2$ and inverting the effect of cell shape on the gradient sensing as $\Delta_{1}^{A}(s) \rightarrow-\Delta_{1}^{A}(s)$ and $\Delta_{1}^{D}(s) \rightarrow-\Delta_{1}^{D}(s)$. Therefore, in this article, we only study the case with $f_{s}>0$.

The functions $\Delta_{1}^{A}(s)$ and $\Delta_{1}^{D}(s)$ represent the dependence of the bias amplitude and dispersion, respectively, on the cell morphology $\boldsymbol{S}$. When the steepness of the chemoattractant gradient is sufficiently large or the concentration of the chemoattractant $C_{0}$ is comparable to the dissociation constant of the receptor $K_{d}=k_{d} / k_{a}$, $\left|A^{0} \Delta_{1}^{A}(s)\right|$ is large. When the steepness is sufficiently small or the concentration $C_{0}$ is much lower or higher than 
$K_{d},\left|D_{q}^{0} \Delta_{1}^{D}(s)\right|$ becomes large. This is because fluctuations in the spatial distribution of the bound chemoattractant molecules on the receptors and the dispersion $D_{q}^{0}$ become large when $C_{0}$ is much lower or higher than $K_{d}$.

The dependences of $A$ and $D_{q}$ on the cell shape have been studied previously $[14,15]$ by considering the reaction between receptors and chemoattractant molecules. When the signal-to-noise ratio in the estimation of the gradient direction is high, the bias amplitude $A$ depends on the cell orientation $\theta_{s}$, whereas, when it is low, the estimation in the direction $\psi$ depends on $\theta_{s}$ as illustrated in fig. 1(c), and thus the noise strength $D_{q}$ depends on $\theta_{q}-\theta_{s}$.

\section{Numerical results}

In this section, we show the numerical results for eq. (13). For $\Delta_{0}^{A}(s)$ and $\Delta_{0}^{D}(s)$ in eqs. (14) and (16), we suppose $\Delta_{0}^{A}(s)=\Delta_{0}^{D}(s)=0$. For $\Delta_{1}^{A}(s)$ and $\Delta_{1}^{D}(s)$, we consider two cases: the linear case $\Delta_{1}^{A}(s)=\mu s$ and $\Delta_{1}^{D}(s)=$ $\sigma s$, and the saturated case $\Delta_{1}^{A}(s)=\mu s /(1+|\mu s|)$ and $\Delta_{1}^{D}(s)=\sigma s /(1+|\sigma s|)$ with constants $\mu$ and $\sigma$. From the requirements that the left-hand side in eq. (15) should be non-negative and that the bias amplitude $A\left(s, \theta_{s}\right)$ in eq. (14) may also be positive, the saturated forms are more realistic. For the numerical calculation of eq. (13), we first changed the variables from $\left(s, \theta_{s}\right)$ to $\left(S_{11}, S_{12}\right)$ as $s=2 \sqrt{S_{11}^{2}+S_{12}^{2}}$ and $\tan \left(2 \theta_{s}\right)=S_{12} / S_{11}$. The Euler scheme was employed with a time increment $\Delta t=0.001$. The discretization of the variables were set to $\Delta \theta_{q}=$ $(2 \pi) / 64, \Delta S_{11}=1 / 64$ and $\Delta S_{12}=1 / 64$. After the nondimensionalization $t \rightarrow \tau_{q} t$, the dimensionless parameter values are given by $A^{0} /\left(f_{q} \tau_{R}\right)=1.0, \tau_{q} E=1.0$ and $\tau_{q} f_{s}=1.0$. We obtained the $\operatorname{PDF} P\left(\theta_{q}, t\right)$ by integrating $P\left(\theta_{q}, S_{11}, S_{12}, t\right)$ with respect to $S_{11}$ and $S_{12}$. Without anisotropy $(\mu=\sigma=0)$, the stationary distribution is given by the $\mathrm{CN}$ in eq. (9). We considered the parameter range $0 \leq \mu \leq 2$ and $-1.4 \leq \sigma \leq 0.7$ for the linear case, and $-5 \leq \mu \leq 5$ and $-5 \leq \sigma \leq 5$ for the saturated case. We confirmed that, within these ranges, the PDFs asymptotically settled into the same stationary distribution, independent of the initial conditions.

The stationary PDFs $P_{s}\left(\theta_{v}\left(=\theta_{q}\right)\right)$ of $P\left(\theta_{q}, t\right)$ are shown in figs. 2(a)-(c) for both the linear case (left) and saturated case (right). The PDFs show deviations from the $\mathrm{CN}$ for $\mu \neq 0$ and $\sigma \neq 0$. Therefore, the cell morphology dynamics can modulate the distributions of the cell migration directions for both linear and saturated cases. The tendencies of the modulation of the distributions do not depend on whether we use linear or saturated functions.

In figs. 3(a) and (b), we also plotted the first and second moments of stationary distributions $P_{s}\left(\theta_{v}\right)$, respectively. Figure 3 shows that the dependence of the two moments on $\mu$ is small for $\sigma=0$. For $\mu=0$, the two moments increase with increasing $\sigma$ for $\sigma>0$.

In summary, the cell shape dynamics can modulate the probability density function of migration directions from the circular normal distribution.
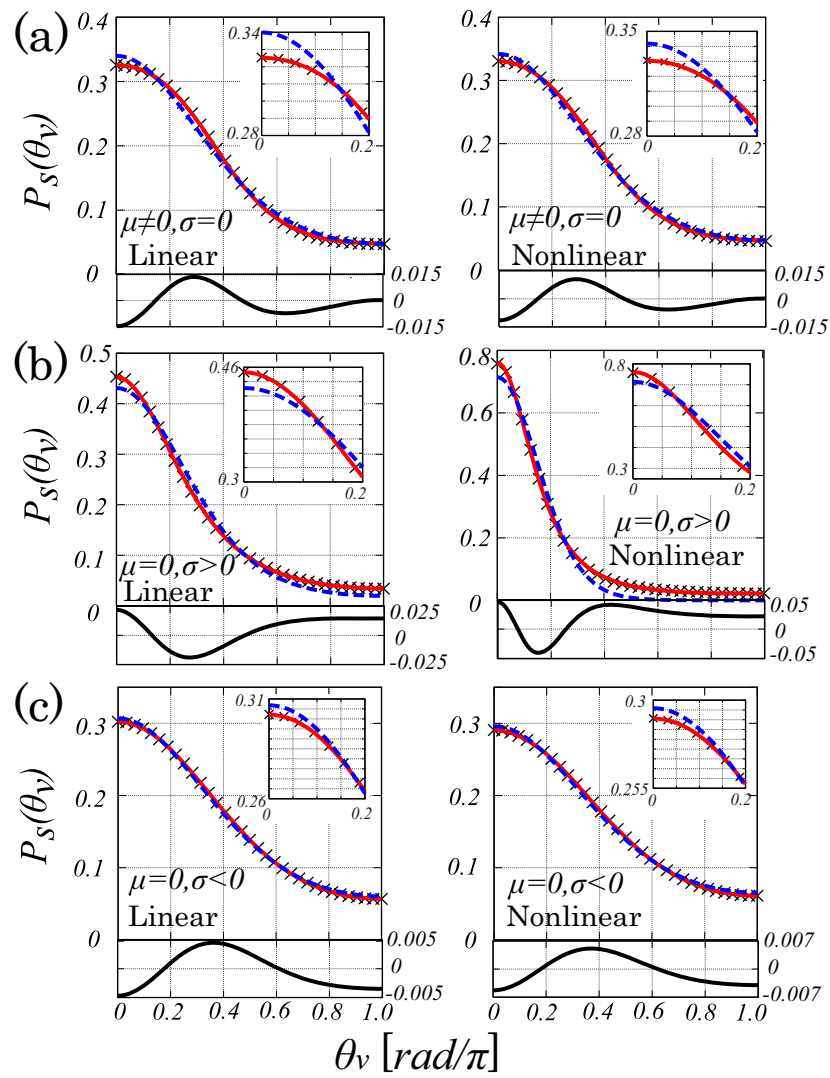

Fig. 2. (Color on-line). (a)-(c) Stationary distributions $P_{s}\left(\theta_{v}\right.$ $\left.\left(=\theta_{q}\right)\right)$. The theoretical curves (red solid line) and the circular normal distributions (blue broken line) are fitted to the numerical results (crosses) by a nonlinear least-square fit. For the theoretical curve, eq. (25) in (a), eq. (28) in (b), and eq. (29) in (c) are used. As the fitting parameters for the theoretical curves and the $\mathrm{CN}$, we take $\kappa^{\prime}$ or $\kappa^{\prime \prime}, \omega$ or $\lambda$ and $\kappa$, respectively, and the overall normalization constants. For numerical simulations, we use the linear functions $\Delta_{1}^{A}(s)=\mu s$ and $\Delta_{1}^{D}(s)=\sigma s$ (left) and the saturated functions $\Delta_{1}^{A}(s)=\mu s /(1+|\mu s|)$ and $\Delta_{1}^{D}(s)=\sigma s /(1+|\sigma s|)$ (right). The parameter values are (a) $\mu=2.0$ and $\sigma=0.0$ for left, and $\mu=5.0$ and $\sigma=0.0$ for right, (b) $\mu=0.0$ and $\sigma=0.7$ for left, and $\mu=0.0$ and $\sigma=5.0$ for right, (c) $\mu=0.0$ and $\sigma=-1.4$ for left, and $\mu=0.0$ and $\sigma=-5.0$ for right. The other parameter values are indicated in the text. Insets show magnifications of the peaks. The lower panels show the differences between the theoretical distributions and the fitted circular normal distributions (black solid lines, right axes). In each graph, the scaled maximum fitting error $\max _{k}\left[\left(P_{k}-f\left(\theta_{v k}\right)\right) / P_{k}\right]$ with the $k$-th data points $\left(\theta_{v k}, P_{k}\right)$ and fitting curve $f\left(\theta_{v}\right)$ for the $\mathrm{CN}$ fitting is ca. 10 times larger than the theoretical curve fitting. In particular, in (b) right, the scaled fitting error reaches $\sim 1.0$ for the $\mathrm{CN}$ fitting, whereas they are less than 0.10 for the theoretical curve.

\section{Analytical explanation of the stationary distributions}

In this section, we study the stationary distributions for the deformable cell, and their deviations from the $\mathrm{CN}$, by considering eq. (13) analytically. 
(a)

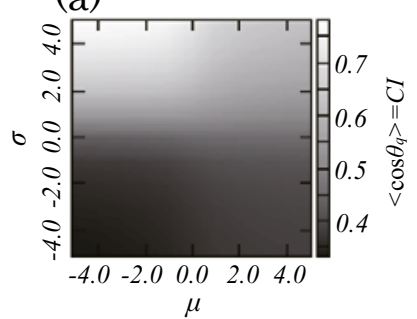

(b)

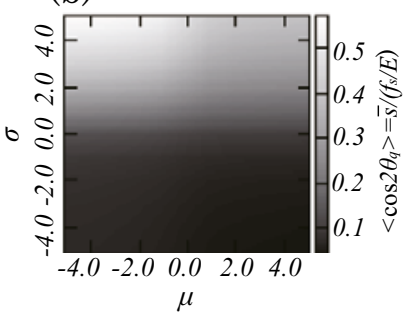

Fig. 3. (a) First and (b) second moments of stationary distributions of migration and polarity directions. In both (a) and (b), we use the saturated functions $\Delta_{1}^{A}(s)=\mu s /(1+|\mu s|)$ and $\Delta_{1}^{D}(s)=\sigma s /(1+|\sigma s|)$. The other parameter values are indicated in the text.

\subsection{Calculation procedure}

By integrating eq. (13) with respect to $s$ and $\theta_{s}$, we obtained

$$
\frac{\partial P\left(\theta_{q}, t\right)}{\partial t}=\frac{\partial}{\partial \theta_{q}}\left[\overline{c_{q}} P\left(\theta_{q}, t\right)\right]+\frac{\partial^{2}}{\partial \theta_{q}^{2}}\left[\overline{D_{q}} P\left(\theta_{q}, t\right)\right],
$$

where over-lines indicate the conditional average with respect to $s$ and $\theta_{s}$ for a given $\theta_{q}$ and $t$. This conditional average can be obtained by interpolating the values in the limits $E \rightarrow \infty$ and $E \sim 0$ with a given value of $f_{s} / E$ (fig. 4(a)).

For $E \rightarrow \infty$, since the deformation $S$ is completely relaxed for a given $\theta_{q}$, we obtained $\theta_{s}=\theta_{q}(\bmod \pi)$ and $s=$ $s_{\infty} \equiv f_{s} / E$ from eq. (12). Substituting these into eq. (14), the average amplitude $A$ of the bias and the diffusion constant $D_{q}\left(\theta_{q}, s, \theta_{s}\right)$ are

$$
\begin{aligned}
& \overline{A\left(s, \theta_{s}\right)}=\bar{A}_{\infty} \equiv A^{0}\left[1+\Delta_{0}^{A}\left(s_{\infty}\right)-\Delta_{1}^{A}\left(s_{\infty}\right) \cos 2 \theta_{q}\right], \\
& \overline{D_{q}\left(\theta_{q}, s, \theta_{s}\right)}=\overline{D_{q_{\infty}}} \equiv D_{q}^{0}\left[1+\Delta_{0}^{D}\left(s_{\infty}\right)-\Delta_{1}^{D}\left(s_{\infty}\right)\right] .
\end{aligned}
$$

For $E \sim 0, s$ and $\theta_{s}$ can be obtained, assuming that $\theta_{q}$ obeys the equilibrium distribution. Under such a condition, from eq. (12) we have $\theta_{s} \sim 0$ and

$$
s \sim \bar{s} \equiv \frac{f_{s}}{E}\left\langle\cos 2 \theta_{q}\right\rangle_{\theta_{q}},
$$

leading to

$$
\begin{aligned}
& \overline{A\left(s, \theta_{s}\right)}=\bar{A}_{0} \equiv A^{0}\left[1+\Delta_{0}^{A}(\bar{s})-\Delta_{1}^{A}(\bar{s})\right], \\
& \overline{D_{q}\left(\theta_{q}, s, \theta_{s}\right)}=\overline{D_{q_{0}}} \equiv D_{q}^{0}\left[1+\Delta_{0}^{D}(\bar{s})-\Delta_{1}^{D}(\bar{s}) \cos 2 \theta_{q}\right] .
\end{aligned}
$$

The averaged magnitude of the persistent deformation $\bar{s}$ is determined self-consistently from the resulting distribution of the polarity direction $\theta_{q}$.

To connect these limits, we introduce a constant $p$, which decreases monotonically from $p=1$ to $p=0$ as $E$

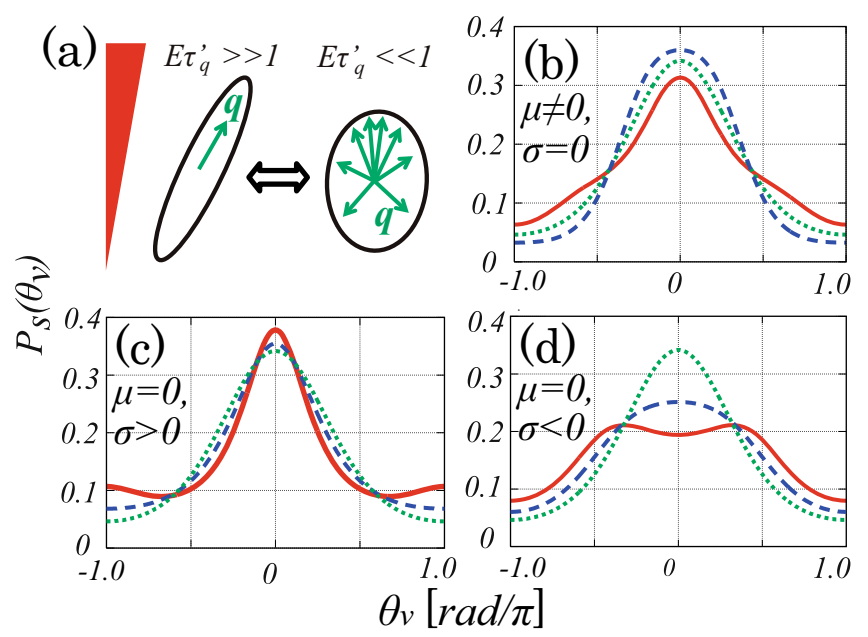

Fig. 4. (Color on-line). (a) The different limits for the present interpolation. (b)-(d) The stationary distributions of $\theta_{v}=\theta_{q}$, with analytical results for eq. (25) (b), eq. (28) (c), and eq. (29) (d). Parameter values are given as follows: (b) $\kappa^{\prime}=1$ and $\mu=0$ (green dotted line), 0.1 (blue broken line), -0.1 (red solid line), (c) $\kappa^{\prime \prime}=1$ and $\lambda=0$ (green dotted line), 0.4 (blue broken line), 0.7 (red solid line), (d) $\kappa^{\prime \prime}=1$ and $\lambda=0$ (green dotted line), 0.4 (blue broken line), 0.7 (red solid line).

decreases from $E \rightarrow \infty$ to $E \rightarrow 0$. Using this constant, the interpolation formulae are approximately given by

$$
\begin{aligned}
\overline{A\left(s, \theta_{s}\right)} & \approx p \bar{A}_{\infty}+(1-p) \bar{A}_{0}, \\
\overline{D_{q}\left(\theta_{q}, s, \theta_{s}\right)} & \approx p{\overline{D_{q_{\infty}}}}+(1-p){\overline{D_{q_{0}}}} .
\end{aligned}
$$

The stationary distributions $P\left(\theta_{q}, t\right)$ can be approximately obtained by substituting eqs. (23) and (24) with $\overline{c_{q}}=\left[f_{q} \overline{A\left(s, \theta_{s}\right)} \sin \theta_{q}\right] / 2$ into eq. (17). In the following subsections, we consider the two different cases.

\subsection{Case A: Bias A depends on the cell morphology}

We first consider the case where $\Delta_{0}^{D}(s)=\Delta_{1}^{D}(s)=0$ and $\Delta_{0}^{A}(s) \neq 0, \Delta_{1}^{A}(s) \neq 0$; the bias amplitude in the direction estimation shows a dependence on the cell orientation as well as the shape, whereas the dispersion in the gradient sensing does not. Such a situation is found when the chemoattractant concentration is comparable to the dissociation constant $K_{d}$, i.e., in the high accuracy limit of gradient estimation. The stationary distribution is obtained as

$$
P_{s}\left(\theta_{q}\right) \propto \exp \left[\left(\kappa^{\prime}+3 \omega\right) \cos \theta_{q}-\omega \cos 3 \theta_{q}\right],
$$

as shown in fig. 4(b), where $\kappa^{\prime}$ is the concentration parameter and $\omega$ characterizes the deviation from the $\mathrm{CN}$, given by

$$
\begin{aligned}
& \kappa^{\prime}=\frac{A^{0}}{f_{q} \tau_{R}}\left\{1+p \Delta_{0}^{A}\left(s_{\infty}\right)+(1-p)\left[\Delta_{0}^{A}(\bar{s})-\Delta_{1}^{A}(\bar{s})\right]\right\} \\
& \omega=\frac{p A^{0} \Delta_{1}^{A}\left(s_{\infty}\right)}{6 f_{q} \tau_{R}}
\end{aligned}
$$


Figure 2(a) shows good agreement between eq. (25) (red solid line) and the fitted numerical results (crosses) with fitting parameters $\kappa^{\prime}$ and $\omega$. We also plot the CN distribution obtained by the fit (blue broken lines), which was narrower than the numerical results and the analytical results eq. (25).

The parameter $\omega$, associated with the deviation from the $\mathrm{CN}$, is estimated as $\omega \sim p \Delta_{1}^{A}\left(s_{\infty}\right)$. Thus, when the cell shape immediately follows the internal polarity with sufficiently large $E$ (fig. 4(a) left), the deviation in the distribution becomes large.

\subsection{Case B: Diffusion constant $D_{q}$ depends on the cell morphology}

We next consider the case where $\Delta_{0}^{A}(s)=\Delta_{1}^{A}(s)=0$ and $\Delta_{0}^{D}(s) \neq 0, \Delta_{1}^{D}(s) \neq 0$, that is, the bias amplitude does not show a dependence on the cell orientation, whereas the dispersion does. Such a situation is found when the chemoattractant concentration is much smaller or much larger than the dissociation constant $K_{d}$, i.e., in the low accuracy limit of gradient estimation. In this case, for $\Delta_{1}^{D}(\bar{s})>0$, we have

$$
P_{s}\left(\theta_{q}\right) \propto \frac{\exp \left[\kappa^{\prime \prime} \frac{1-\lambda^{2}}{\lambda} \operatorname{arctanh}\left(\lambda \cos \theta_{q}\right)\right]}{1-\left(\lambda \cos \theta_{q}\right)^{2}},
$$

as shown in fig. 4(c), whereas for $\Delta_{1}^{D}(\bar{s})<0$

$$
P_{s}\left(\theta_{q}\right) \propto \frac{\exp \left[\kappa^{\prime \prime} \frac{1+\lambda^{2}}{\lambda} \arctan \left(\lambda \cos \theta_{q}\right)\right]}{1+\left(\lambda \cos \theta_{q}\right)^{2}},
$$

as shown in fig. $4(\mathrm{~d})$. Here, $\kappa^{\prime \prime}$ is the concentration parameter, and $\lambda$ is the sharpness parameter ${ }^{1}$, given by

$$
\begin{aligned}
\kappa^{\prime \prime}= & \frac{A^{0}}{f_{q} \tau_{R}}\left\{1+p\left[\Delta_{0}^{D}\left(s_{\infty}\right)-\Delta_{1}^{D}\left(s_{\infty}\right)\right]\right. \\
& \left.+(1-p)\left[\Delta_{0}^{D}(\bar{s})-\Delta_{1}^{D}(\bar{s})\right]\right\}^{-1} \\
\lambda= & \sqrt{2(1-p)\left|\Delta_{1}^{D}(\bar{s})\right|}\left\{1+p\left[\Delta_{0}^{D}\left(s_{\infty}\right)-\Delta_{1}^{D}\left(s_{\infty}\right)\right]\right. \\
& \left.+(1-p)\left[\Delta_{0}^{D}(\bar{s})+\Delta_{1}^{D}(\bar{s})\right]\right\}^{-1 / 2} .
\end{aligned}
$$

The sharpness parameter $\lambda$ characterizes the deviation of $P_{s}\left(\theta_{q}\right)$ from the CN. In figs. 2(b) and (c), eqs. (28) and (29) are shown (red solid lines) with the numerical results (crosses), respectively, demonstrating good agreement. The parameter values of $\kappa^{\prime \prime}$ and $\lambda$ were obtained from the fit. For each case, we also fitted the CN to the numerical results (blue broken lines) showing characteristic deviations. For $\Delta_{1}^{D}(\bar{s})>0$, the CN distribution is broader than eq. (28), but sharper than eq. (29) for $\Delta_{1}^{D}(\bar{s})<0$.

\footnotetext{
${ }^{1}$ In eq. (29), $\lambda$ means the broadness rather than the sharpness. However, in this article, we use the terminology "sharpness" for both cases for convenience.
}

Note that the sharpness $\lambda$ is estimated as $\lambda \sim \mid(1-p)$ $\left.\Delta_{1}^{D}(\bar{s})\right|^{1 / 2}$, suggesting that the condition $E \sim 0$ leads to distortions in the PDFs given by eqs. (28) and (29), as described in fig. 4(a) right. This means that $\Delta_{1}^{D}$ results in the anisotropy of the diffusion constant of $\theta_{q}$, giving $D_{q} \sim$ $\left(f_{q}^{2} \tau_{R} / 2\right)\left(1-\Delta_{1}^{D}(\bar{s})\right)$ for $\theta_{q} \sim 0, \pi$, and $D_{q} \sim\left(f_{q}^{2} \tau_{R} / 2\right)$ $\left(1+\Delta_{1}^{D}(\bar{s})\right)$ for $\theta_{q} \sim \pm \pi / 2$. Thus, for $\Delta_{1}^{D}(\bar{s})>0$, when the internal polarity is directed perpendicular to the gradient, the polarity direction is more variable. We note that this anisotropy cannot be neglected even for a shallow gradient or low concentration of chemoattractants, because $\bar{s}$, and hence $\Delta_{1}^{D}(\bar{s})$ in eq. (22), is not sufficiently small, as shown below. This exhibits a strong contrast to the case of a circular cell as shown in eq. (7). Because the characteristic response time $f_{q}^{-1}$, which is tens of seconds, is much larger than $\tau_{R} \sim 1$ second, $\kappa^{\prime \prime}$ in eq. (30) is not small. Consequently, the distribution becomes more leptokurtic, i.e., more concentrated around the mean. As a result, $\bar{s}$ becomes large as calculated from eq. (20). Furthermore, $\bar{s}$ becomes even larger for the case that $f_{s}>0$ and $\Delta_{1}^{D}(s)>0$ for any $s>0$, considering eqs. (20), (28) and (31) self-consistently as discussed in sect. 6 in more detail. We also note that the present interpolation approximation neglects the correlation between fluctuations in $s$ and $\theta_{s}$ like the mean-field approximation.

\subsection{Summary of this section}

In this section, we have derived the expressions of stationary distributions of polarity directions. We have found that the stationary distributions of polarity directions show deviations from the circular normal distribution.

We first considered the case when the cell morphology affects the bias amplitude $A$ in estimated directions of the chemical gradient. Such a situation can be found when the steepness of a chemoattractant gradient is sufficiently large and the chemoattractant concentration is comparable to the dissociation constant $K_{d}$ of the receptor. In this situation, the distribution $P_{s}\left(\theta_{q}\right)$ is given by eq. (25) and fig. 4(b). The deviation in the stationary PDF is pronounced when the cell deforms quickly to the polarity direction as described in fig. 4(a) left.

We also considered the case when the dispersion $D_{q}$ can be dependent on the cell morphology. Such a situation can be found when the cells are subjected to a faint gradient. When the dispersion $D_{q}$ is enhanced along the direction parallel to the cell orientation (fig. 1(c)), the stationary distribution is sharpened as shown by eq. (28) and fig. $4(\mathrm{c})$. Conversely, when $D_{q}$ is strengthened along the direction perpendicular to the cell orientation, the stationary distribution is broadened as shown by eq. (29) and fig. 4(d). We found that the deviation becomes strong when the elliptical cell shape is persistently oriented to the chemical gradient with its slow deformation speed as described in fig. 4(a) right.

All of these tendencies are consistent with the numerical results shown in fig. 2 . 


\section{Summary and discussion}

\subsection{Summary}

In this article, we have developed a theory to calculate the statistics of migration directions of chemotactic eukaryotic cells which include shape deformations. We consider the influence of gradient sensing on deformable self-propelled objects, which has not been studied so far. We have derived the possible stationary distributions of migration directions for elliptically deformable cells, both numerically and analytically, as shown by eqs. (25), (28), and (29). We found that the stationary distributions show deviations from the circular normal distribution qualitatively due to the coupling between the gradient sensing ability and shape deformation.

\subsection{Interdependence of the deformation and chemotactic ability}

To further clarify the influence of this coupling on the stationary distribution of migration directions, we consider the interdependence of the deformation $\bar{s}$ and sharpness $\lambda$ of the directional distributions. In this paragraph, for simplicity, we assume $f_{s}>0, \Delta_{0}^{A}(s)=\Delta_{0}^{D}(s)=0, \Delta_{1}^{A}(s)=$ $\mu s /(1+|\mu s|)$ and $\Delta_{1}^{D}(s)=\sigma s /(1+|\sigma s|)$. For the case when $\mu \sim 0$, the deformation $\bar{s}$ is determined by the PDF as shown by eq. (20), whereas the PDFs are functions of $\bar{s}$ as is evidenced in eqs. (28) and (29) with eqs. (30) and (31). For the $\sigma>0$ case, both the deformation $\bar{s}$ and sharpness $\lambda$ are positively interdependent so that both are enhanced by one another. The probability $P_{F} \sim 1+\left(A^{0} / f_{q} \tau_{R}\right)$ that the polarity $\boldsymbol{q}$ directs to the true gradient direction is larger than the probability $P_{B} \sim 1-\left(A^{0} / f_{q} \tau_{R}\right)$ with which the polarity directs to the opposite direction. From the difference between $P_{F}$ and $P_{B}$, we can estimate the contribution of the variation in the diffusion constant $D_{q}$ on the increase in the concentration parameter $\kappa$ and the chemotaxis index $\mathrm{CI} \equiv\left\langle\cos \theta_{q}\right\rangle$. For instance, for small bias $A^{0}$ and deformation $\bar{s}$, considering the variation $\Delta D_{q} \sim D_{q}^{0} \Delta_{1}^{D}(s)$ in $D_{q}$, the increase can be estimated as $\Delta \kappa \equiv \kappa^{\prime \prime}-\kappa \sim\left[P_{F} \Delta D_{q} / D_{q}^{0}-P_{B} \Delta D_{q} / D_{q}^{0}\right] / 2 \sim$ $\left\{A^{0} /\left(f_{q} \tau_{R}\right)\right\}\left[p \Delta_{1}^{D}\left(s_{\infty}\right)+(1-p) \Delta_{1}^{D}(\bar{s})\right]$. This estimation is indeed consistent with the first-order terms of eq. (30) with respect to $\Delta_{1}^{D}$. In contrast, for the $\sigma<0$ case, the interdependence shows a negative effect so that both the deformation $\bar{s}$ and the sharpness $\lambda$ in the PDF are small. On the other hand, for the case when $\mu \neq 0$ and $\sigma=0$, $\bar{s}$ is determined by the ratio between the strength of the driving force and the elastic constant, $s_{\infty}=f_{s} / E$. Hence, in this case, no effective interdependence is present. These effects are demonstrated in figs. 2 and 3. For example, for $\mu=0, \sigma=5$ we have $\mathrm{CI} \sim 0.73$ and $\bar{s} \sim 0.49$, which are much larger than $\mathrm{CI} \sim 0.45$ and $\bar{s} \sim 0.11$ for $\mu=\sigma=0$ and $\mathrm{CI} \sim 0.36$ and $\bar{s} \sim 0.053$ for $\mu=0, \sigma=-5$. Our findings described in this paragraph are schematically summarized in fig. 5 .

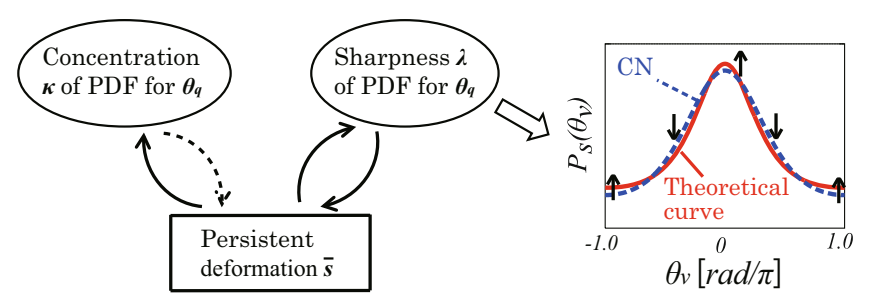

Fig. 5. (Color on-line). The schematic summary of our main findings. At the leading order of the concentration parameter $\kappa$, the persistent deformation $\bar{s}$ and sharpness $\lambda$ influence each other, and the resulting $\bar{s}$ influences $\kappa$ (solid curved arrows in left). The influence of $\kappa$ on $\bar{s}$ arises from only its nonlinear order (broken curved arrow in left). In particular, for $f_{s}>0$ and $\Delta_{1}^{D}>0$, or $f_{s}<0$ and $\Delta_{1}^{D}<0$, this mechanism enhances the sharpness $\lambda$, which leads to the large deviation (solid straight arrows in right) of the stationary distribution of migration direction $\theta_{v}$ from the circular normal distribution $(\mathrm{CN})$ as illustrated in the right figure.

\subsection{Insight into the motility and sensing ability of chemotactic cells}

In this subsection, we discuss the use of our theory to gain insight into the motile and sensing abilities of eukaryotic cells based on the chemotaxis of Dictyostelium cells. The distribution of migration directions of Dictyostelium cells has been measured experimentally [3]. This distribution is sharper than the $\mathrm{CN}$, which can be fitted well using eq. (28) [3]. Together with our results, the characteristic distribution obtained experimentally can be explained by polarity and cell deformation. Interestingly, in ref. [3], eq. (28) was derived from a different model, in which the direction of motion depends on the cell's estimation of gradient direction and its steepness. Then, by assuming that the stochastic fluctuation in the estimation is small, a linear Langevin equation with multiplicative noise was derived. This noise resulted in the deviation in the stationary distribution from the $\mathrm{CN}$ and the nonzero value in the sharpness parameter $\lambda[3]$. A sharp distribution, as in eq. (28), was then derived by further assuming that the direction of cell migration turns to the gradient stronger as the estimated steepness increases [3]. In our case, the multiplicative noise in eq. (13) was derived from the deterministic effect of the cell shape $\boldsymbol{S}$ rather than the linear approximation of Gaussian noise due to directional estimation. Hence, our results are applicable to the situations when the noise is relatively large, such as under a shallow and low concentration gradient. Therefore, we expect, based on our results, that the migration direction, even under a faint gradient, follows the same tendency as in eq. (28).

Under a shallow and low concentration gradient (figs. 4(c) and (d)), $A^{0} \Delta_{1}^{A}(s) \sim 0$, and the weak bias toward the gradient direction is independent of the cell orientation $\theta_{s}$ on average. From eq. (16) we see that the difference in the sign of $\Delta_{1}^{D}(\bar{s})$ results in a difference in the dependence of the variability of the internal polarity $\theta_{q}$ on the cell orientation $\theta_{s}$. By considering the maximum likelihood estimation (MLE) of the gradient direction, we re- 
cently found that $\Delta_{1}^{D}(s)>0$ if the MLE is performed only for the gradient direction given a presupposed steepness, whereas $\Delta_{1}^{D}(s)<0$ if the MLE is performed when neither steepness nor direction is presupposed [15]. By relating our results to the experimental result mentioned above, the case of $\Delta_{1}^{D}(s)>0$ seems more probable, implying that, in Dictyostelium cells, the limit of the gradient sensing ability may not be given by the MLE for both steepness and direction. Together with the above discussion on the interdependence of $\bar{s}$ and $\lambda$, this implication on the sensing ability seems to be consistent with the highly elongated shapes of Dictyostelium cells under a cAMP gradient [24]. A mechanism that biases the internal polarity to the cell shape orientation may also be possible, considering the experimental fact that the non-uniform distribution of some intracellular processes tends to orient to the leading edge of the cell. It has been suggested that F-actin at the cell tip may activate some signaling molecules, which could give such a mechanism.

\subsection{Outlook}

Though we have investigated the influence of the cell morphology only on gradient sensing in this article, our mathematical formulation based on the Langevin equation for the tensor representation in eq. (11) and the FokkerPlanck equation in eq. (13) are applicable to other types of couplings, such as the direct coupling between the polarity $\boldsymbol{q}$ and cell shape $\boldsymbol{S}$ in eq. (25) [19,20,23]. We can also consider the case when the $x$-component of $\langle\boldsymbol{f}(\boldsymbol{S})\rangle$ in eq. (11) depends on the cell morphology $\boldsymbol{S}$. Moreover, by introducing the appropriate interactions between cells, such as cell adhesions, contact inhibitions and the effect of secretion of chemoattractant molecules from each cell into the chemical field, we will be able to investigate collective behaviors of eukaryotic cells.

Our theory is based on the central limit theorem as in eq. (3), so the resultant stationary distributions (25), (28), and (29) are robust for any species of eukaryotic cells as long as $\tau_{P} \gg \tau_{R}$. We expect that our results are applicable to chemotactic migration of other kinds of eukaryotic cells.

This work has been supported by KAKENHI (23111531).

\section{Appendix A. The equation of the polarity vector $q$ with cell morphology}

In this appendix, we note the details of eq. (11) of the presented cell motility model with cell deformation.

The Fokker-Planck equation for $\theta_{q}$ given by eq. (13) with eqs. (14) and (16) corresponds to the Langevin equation for $\boldsymbol{q}$ given by eq. (11) with the averaged driving force

$$
\langle\boldsymbol{f}(\boldsymbol{S}(t))\rangle=\left\{1+\Delta_{0}^{A}(s)-\Delta_{1}^{A}(s)\left[2(\boldsymbol{n} \cdot \hat{\boldsymbol{f}})^{2}-1\right]\right\}\left\langle\boldsymbol{f}^{0}\right\rangle
$$

and the dispersion

$$
\boldsymbol{\xi}(t, \boldsymbol{S}(t))=\boldsymbol{\xi}_{\|}(t, \boldsymbol{S}(t))+\boldsymbol{\xi}_{\perp}(t, \boldsymbol{S}(t)),
$$

where

$$
\boldsymbol{\xi}_{\|}(t, \boldsymbol{S})=(g(s)+h(s)) W_{1}(t) \boldsymbol{n}
$$

and

$$
\boldsymbol{\xi}_{\perp}(t, \boldsymbol{S})=(g(s)-h(s)) W_{2}(t) \boldsymbol{n}_{\perp} .
$$

Here, $\left\langle\boldsymbol{f}^{0}\right\rangle$ is given by eq. $(4), W_{i}(t)$ is white Gaussian noise with $\left\langle W_{i}(t)\right\rangle=0$ and $\left\langle W_{i}(t) W_{j}\left(t^{\prime}\right)\right\rangle=\delta_{i j} \delta\left(t-t^{\prime}\right)$, $\boldsymbol{n}_{\perp}$ is a unit vector perpendicular to $\boldsymbol{n}$, i.e. the unit vector parallel to the minor (short) axis of the cell, $\hat{\boldsymbol{f}} \equiv(0,1)$ is the unit vector parallel to the true gradient direction, and the functions $g(s)$ and $h(s)$ are given as the solution of $g(s)^{2}+h(s)^{2}=f_{q}^{2} \tau_{R}\left[1+\Delta_{0}^{D}(s)\right]$ and $g(s) \cdot h(s)=$ $f_{q}^{2} \tau_{R} \Delta_{1}^{D}(s) / 2$.

We also comment that, from the general point of view of the coupling of tensors $[19,20,23]$, the influence of the morphology $\boldsymbol{S}$ on the noisy driving force $\langle\boldsymbol{f}(\boldsymbol{S})\rangle+\boldsymbol{\xi}(t, \boldsymbol{S})$ is given by

$$
\langle\boldsymbol{f}(\boldsymbol{S})\rangle=\left(1+a \boldsymbol{S}+b \sum_{i, j=x, y} \hat{f}_{i} S_{i j} \hat{f}_{j}\right)\left\langle\boldsymbol{f}^{0}\right\rangle+O\left(\boldsymbol{S}^{2}\right)
$$

and

$$
\begin{aligned}
\boldsymbol{\xi}(t, \boldsymbol{S})= & \left\{1+c \boldsymbol{S}+d \sum_{i, j=x, y} \hat{f}_{i} S_{i j} \hat{f}_{j}+e(\boldsymbol{S} \hat{\boldsymbol{f}}) \hat{\boldsymbol{f}}\right. \\
& \left.+r \hat{\boldsymbol{f}}(\boldsymbol{S} \hat{\boldsymbol{f}}) \cdot+u \hat{\boldsymbol{f}}\left(\sum_{i, j=x, y} \hat{f}_{i} S_{i j} \hat{f}_{j}\right) \hat{\boldsymbol{f}} \cdot\right\} \boldsymbol{\xi}^{0}(t) \\
& +O\left(\boldsymbol{S}^{2}\right)
\end{aligned}
$$

with the coefficients $a, b, c, d, e, r$, and $u$ at the lowest order with respect to $\boldsymbol{S}$. The nonlinear order terms can be obtained by the same strategy. The terms with $\Delta_{1}^{A}(s)$ in the averaged driving force $\langle\boldsymbol{f}\rangle$ and $\Delta_{1}^{D}(s)$ in the noise term $\boldsymbol{\xi}$ correspond to the terms with the coefficient $b$ in eq. (A.5) and $c$ in eq. (A.6), respectively, and the nonlinear order terms with respect to $S$. The terms with $\Delta_{0}^{A}(s)$ and $\Delta_{0}^{D}(s)$ come from the nonlinear order terms.

Open Access This is an open access article distributed under the terms of the Creative Commons Attribution License (http://creativecommons.org/licenses/by/3.0), which permits unrestricted use, distribution, and reproduction in any medium, provided the original work is properly cited.

\section{References}

1. Y.T. Maeda, J. Inoue, M.Y. Matsuo, S. Iwaya, M. Sano, PLoS ONE 3, e3734 (2008).

2. A. Mogilner, K. Keren, Curr. Biol. 19, R762 (2009).

3. B. Hu, D. Fuller, W.F. Loomis, H. Levine, W. Rappel, Phys. Rev. E 81, 031906 (2010).

4. H. Takagi, M. Sato, T. Yanagida, M. Ueda, PLoS ONE 3, e2648 (2008).

5. L. Li, E.C. Cox, H. Flyvbjerg, Phys. Biol. 8, 046006 (2011).

6. P.R. Fisher, R. Merkl, G. Gerish, J. Cell. Biol. 108, 973 (1989). 
7. S.M. Nadkarni, H.U. Bödeker, C. Beta, A. Bae, C. Eranck, W.-J. Rappel, W.F. Loomis, E. Bodenschatz, Euro. J. Cell Biol. 85, 981 (2006).

8. L. Li, S.F. Norrelykke, E.C. Cox, PLoS ONE 3, e2093 (2008).

9. W. Chen, M. Adler, A. Groisman, H. Levine, W. Rappel, W.F. Loomis, Proc. Natl. Acad. Sci. U.S.A. 107, 9656 (2010).

10. P.J.M. Haastert, M. Postma, Biophys. J. 93, 1787 (2007).

11. M. Postma, J. Roelofs, J. Goedhart, H.M. Loovers, A.J.W.G. Visser, P.J.M. Haastert, J. Cell Sci. 117, 2925 (2004).

12. Y. Arai, T. Shibata, S. Matsuoka, M.J. Sato, T. Yanagida, M. Ueda, Proc. Natl. Acad. Sci. U.S.A. 107, 12399 (2010).

13. T. Shibata, M. Nishikawa, S. Matsuoka, M. Ueda, J. Cell Sci. 125, 5138 (2012).

14. B. Hu, W. Chen, W. Rappel, H. Levine, Phys. Rev. E 83, 021917 (2011).
15. A. Baba, T. Hiraiwa, T. Shibata, Phys. Rev. E 86, 060901(R) (2012).

16. M. Ueda, Y. Sako, T. Tanaka, P. Devreotes, T. Yanagida, Science 294, 864 (2001).

17. M. Ueda, T. Shibata, Biophys. J. 93, 11 (2007).

18. M. Schienbein, H. Gruler, Bull. Math. Biol. 55, 585 (1993).

19. T. Ohta, T. Ohkuma, Phys. Rev. Lett. 102, 154101 (2009).

20. T. Hiraiwa, M.Y. Matsuo, T. Ohkuma, T. Ohta, M. Sano, EPL 91, 20001 (2010).

21. B. Hu, W. Chen, W. Rappel, H. Levine, Phys. Rev. Lett. 105, 048104 (2010).

22. P.-G. de Gennes, J. Prost, The Physics of Liquid Crystals (Oxford University Press, Oxford, 1993).

23. M. Tarama, T. Ohta, Eur. Phys. J. B 83, 391 (2011).

24. B. Meier, A. Zielinski, C. Weber, D. Arcizet, S. Youssef, T. Franosch, J.O. Rädler, D. Heinrich, Proc. Natl. Acad. Sci. U.S.A. 108, 11417 (2011). 